\title{
4G model of final unification - A very brief report
}

\author{
Seshavatharam U.V.S ${ }^{1,2}$ and Lakshminarayana $S^{3}$ \\ ${ }^{1}$ Honorary faculty, I-SERVE, Survey no-42, Hitech city, Hyderabad-84,Telangana, INDIA \\ ${ }^{2}$ Asst. Mgr., QA Dept, DIP-SPUN, Srikalahasthi Pipes Ltd, Srikalahasthi-517641, AP, INDIA \\ ${ }^{3}$ Dept. of Nuclear Physics, Andhra University, Visakhapatnam-03, AP, INDIA \\ Emails: seshavatharam.uvs@gmail.com (and) lnsrirama@gmail.com \\ Orcid numbers : 0000-0002-1695-6037 (and) 0000-0002-8923-772X
}

\begin{abstract}
To understand the mystery of final unification, in our earlier publications, we proposed that, 1) There exist three atomic gravitational constants associated with electroweak, strong and electromagnetic interactions; 2) There exists a strong interaction elementary charge in such a way that, it's squared ratio with normal elementary charge is close to inverse of the strong coupling constant; and 3) Considering a fermion-boson mass ratio of 2.27, quarks can be split into quark fermions and quark bosons. Further, we noticed that, electroweak field seems to be operated by a primordial massive fermion of rest energy $584.725 \mathrm{GeV}$ and hadron masses seem to be generated by a new hadronic fermion of rest energy $103.4 \mathrm{GeV}$. In this context, starting from lepton rest masses to stellar masses, we have developed many interesting and workable relations. With further study, a workable model of final unification can be developed.
\end{abstract}

Key words: Four gravitational constants; Strong nuclear charge; Electroweak fermion; Hadron mass generator; Super symmetry;

\begin{tabular}{|ll|l|}
\hline \multicolumn{2}{|l|}{ Nomenclature } \\
\hline 1) & Newtonian gravitational constant $=G_{N}$ & 9) Strong coupling constant $=\alpha_{s}$ \\
2) Electromagnetic gravitational constant $=G_{e}$ & 10) Elementary charge $=e$ \\
3) Nuclear gravitational constant $=G_{s}$ & 11) Strong elementary charge $=e_{s}$ \\
4) Weak gravitational constant $=G_{W}$ & 12) Mass of proton $=m_{p}$ \\
5) Fermi's weak coupling constant $=G_{F}$ & 13) Mass of electron $=m_{e}$ \\
6) New electroweak fermion $=M_{w f}$ & 14) Fermion-boson mass ratio $=\Psi$ \\
7) Reduced Planck's constant $=\hbar$ & 15) Hadron mass generator $=M_{h f}$ \\
8) & Speed of light $=c$ & \\
\hline
\end{tabular}

\section{Introduction}

Even though celestial objects that show gravity are confirmed to be made up of so many atoms, so far scientists could not find any relation in between gravity and the atomic interactions at quantum gravity level [1,2]. Black hole temperature point of view [3], strong interaction point of view [4-7] and electroweak interaction point of view [8], scientists found very interesting similarities in between gravity and quantum phenomena. Quantum cosmology point of view [9] and nuclear quantum gravity point of view [10-20], we could develop workable ideas, concepts and relations. Super symmetry point of view [21-24], we proposed a method for understanding baryon and meson masses. On a whole, workability is still lagging. It clearly indicates that, there is something wrong in our notion of understanding or there is something missing in developing the unified physical concepts and needs a critical review at fundamental level. In this context, we hope that, electroweak scale $[25,26,27]$ can certainly yield useful stuff.

\section{Basic assumptions}

1) Each atomic interaction is associated with a characteristic gravitational coupling constant.

2) There exists a characteristic electroweak fermion of rest energy [18], $M_{w f} c^{2} \cong 584.725 \mathrm{GeV}$. It can be considered as the zygote of all elementary particles. 
3) Fermi's weak coupling constant $\left(G_{F}\right)$ can be considered as the basic unified coupling constant.

4) There exists a strong interaction elementary charge $\left(e_{\mathrm{s}}\right)$ in such a way that, it's squared ratio with normal elementary charge is close to inverse of the strong coupling constant [28].

5) There exists a hadronic fermion of rest energy $M_{h f} c^{2} \cong 103.4 \mathrm{GeV}$. It can be called as hadron mass generator.

6) Fermion - boson mass ratio is, $\Psi \cong 2.27$.

\section{Characteristic unified relations}

Based on the above points, we propose the following new and workable relations.

$$
\begin{aligned}
& \hbar c \cong G_{w} M_{w f}^{2} \cong \sqrt{G_{F}\left(\frac{c^{4}}{4 G_{w}}\right)} \\
& \Rightarrow \hbar \cong \frac{G_{w} M_{w f}^{2}}{c} \cong \sqrt{\frac{G_{F} c^{2}}{4 G_{w}}}
\end{aligned}
$$

where $\left(\frac{c^{4}}{4 G_{w}}\right) \cong 6.9401 \times 10^{10} \mathrm{~N}$ is the characteristic force associated with electroweak interaction.

$$
\begin{gathered}
m_{e} \cong\left(\frac{G_{w}}{G_{s}}\right) M_{w f} \\
m_{p} \cong\left(\frac{G_{s}}{G_{w}}\right)\left(\frac{G_{s}}{G_{e}}\right) M_{w} \cong\left(\frac{G_{s}^{2}}{G_{w} G_{e}}\right) M_{w f} \\
\frac{M_{w f}}{m_{e}} \cong \frac{G_{w}^{5 / 2} G_{e}^{5 / 3}}{G_{s}^{4} G_{N}^{1 / 6}} \\
\frac{M_{w f}}{m_{p}} \cong \frac{G_{s}^{1 / 2} G_{e}^{1 / 6} G_{N}^{1 / 12}}{G_{w}^{3 / 4}} \\
\frac{m_{p}}{m_{e}} \cong \frac{G_{w}^{13 / 4} G_{e}^{3 / 2}}{G_{s}^{9 / 2} G_{N}^{1 / 4}} \cong \frac{G_{s}^{3}}{G_{w}^{2} G_{e}}
\end{gathered}
$$

\section{Specific unified relations connected with $\left(G_{e}, G_{s}, G_{w}, G_{N}\right)$}

In a semi empirical approach,

$$
\frac{m_{p}}{m_{e}} \cong 2 \pi \sqrt{\frac{4 \pi \varepsilon_{0} G_{e} m_{e}^{2}}{e^{2}}}
$$

$$
\begin{aligned}
& \text { 8A) } \frac{m_{p}}{m_{e}} \cong\left(\frac{G_{s} m_{p}^{2}}{\hbar c}\right)\left(\frac{G_{e} m_{e}^{2}}{\hbar c}\right) \\
& \text { 8B) } \frac{m_{p}}{m_{e}} \cong\left(\frac{e_{s}^{2}}{4 \pi \varepsilon_{0} G_{s} m_{p}^{2}}\right) /\left(\frac{e^{2}}{4 \pi \varepsilon_{0} G_{e} m_{e}^{2}}\right) \\
& \text { 8C) } \frac{e_{s}}{e} \cong \sqrt{\frac{1}{\alpha_{s}}} \cong\left(\frac{G_{s} m_{p}^{2}}{\hbar c}\right) \cong \sqrt{\frac{G_{s} m_{p}^{3}}{G_{e} m_{e}^{3}} \cong\left(\frac{G_{s}^{5}}{G_{e}^{2} G_{w}^{3}}\right)}
\end{aligned}
$$

$$
G_{N} \cong\left(\frac{m_{e}}{m_{p}}\right)^{10} G_{w} \cong \frac{G_{w}^{21} G_{e}^{10}}{G_{s}^{30}}
$$

Based on these relations,

On step-by-step,

1) Based on relation (7), $\left(G_{e}\right)$ can be estimated.

2) Based on relation (8A), $\left(G_{s}\right)$ can be estimated.

3) Based on relation (6), $\left(G_{w}\right)$ can be estimated.

4) Based on relation (9), $\left(G_{N}\right)$ can be estimated

5) Based on relation $(8),\left(\alpha_{s}, e_{s}\right)$ can be estimated.

6) Based on relation (1), $\left(G_{F}\right)$ can be estimated.

Thus, quantitatively,

$$
\begin{gathered}
G_{e} \cong 2.374335 \times 10^{37} \mathrm{~m}^{3} \mathrm{~kg}^{-1} \mathrm{sec}^{-2} \\
G_{s} \cong 3.329561 \times 10^{28} \mathrm{~m}^{3} \mathrm{~kg}^{-1} \mathrm{sec}^{-2} \\
G_{w} \cong 2.909745 \times 10^{22} \mathrm{~m}^{3} \mathrm{~kg}^{-1} \mathrm{sec}^{-2} \\
\frac{G_{N} \cong 6.679855 \times 10^{-11} \mathrm{~m}^{3} \mathrm{~kg}^{-1} \mathrm{sec}^{-2}}{\frac{G_{F} \cong 1.44021048 \times 10^{-62} \mathrm{~J}^{3}}{3}} \\
e_{s} \cong 2.9463591 e \\
\alpha_{S} \cong 0.1151937 \\
\left(\alpha_{s}\right)^{-1} \cong 8.681032
\end{gathered}
$$

5. To understand the integral nature of electron's angular momentum

Without considering the rest mass of proton, Bohr's theory of Hydrogen atom [29] attempts to explain the discrete spectral lines. On a whole, 
a) If hydrogen atom is characterized by its central mass and central charge,

b) If mass of proton is 1836 times heavier than electron,

then, ignoring proton mass in the calculation of emitted spectral lines seems to be a fundamental snag. Probably it may be the root cause of failure of developing a unified model. With our approach, it is possible to show that,

$$
\begin{gathered}
\hbar c \cong\left(\frac{G_{w} G_{e}}{G_{s}}\right) m_{p} m_{e} \\
\hbar \cong\left(\frac{1}{c}\right)\left(\frac{G_{w} G_{e}}{G_{s}}\right) m_{p} m_{e}
\end{gathered}
$$

As per the Bohr's second postulate,

$$
\left(m_{e} v r\right) \cong n \hbar \cong n\left(\frac{1}{c}\right)\left(\frac{G_{w} G_{e}}{G_{s}}\right) m_{p} m_{e}
$$

where, $n=1,2,3,$. .

It can be inferred as,

$$
m_{e}(v r) \cong\left[\left(\frac{1}{c}\right)\left(\frac{G_{w} G_{e}}{G_{s}}\right)\left(n m_{p}\right)\right] m_{e}
$$

Clearly speaking, integral nature of $m_{p}$ i.e. $m_{p}, 2 m_{p}$, $3 m_{p}, . . n m_{p}$, seems to be responsible for the integral nature of electron's angular momentum. This explanation seems to be very natural and very simple.

\section{Interesting outcomes}

1) Mystery of H-bar and integral nature of angular momentum [29] can be understood [30].

2) Four interaction ranges can be understood with a common expression [31].

3) Nuclear stability line can be understood with proton number [18,19,32].

4) Nuclear binding energy can be understood with 3 simple terms having single energy coefficient $[18,19,32]$.

5) Nuclear charge radii can be fitted with a simple formula [13,33].

6) Nuclear magic numbers can be understood with quarks [32].

7) Proton and electron rest masses can be fitted.
8) Neutron and proton rest masses can be fitted [21-24].

9) Based on strong charge conservation and Super Symmetry, fractional charge quarks can be understood [34].

10) Quark fermion and quark boson masses can be estimated [21-24].

11) Baryon and meson masses can be fitted with Fluons and Bluons respectively [31].

12) Charged lepton masses and $3.5 \mathrm{keV}$ galactic photons can be fitted [34,35,36,37].

13) Electroweak particle masses can be fitted[34].

14) Elementary particle melting points can be understood [5,34].

15) Neutron life time can be fitted $[34,38,39,40]$.

16) Characteristic atomic radius can be fitted [41].

17) Stellar mass limits can be understood [19, 34,42].

18) Stellar magnetic dipole moments can be understood [43].

19) Newtonian gravitational constant can be estimated with atomic physical constants $[44,45]$.

20) Electroweak [25-27] and Planck scales can be studied in a unified manner [34].

21) Nature of dark matter [44] can be studied with $585 \mathrm{GeV}$ electroweak fermion [18].

\section{Conclusion}

With further study, research and confirming the existence of the proposed $\left(M_{w f} c^{2}\right)^{ \pm} \cong 584.725 \mathrm{GeV}$, actual essence of final unification can be understood. Microscopic and macroscopic physical constants can be reviewed in a unified manner.

\section{Acknowledgements}

Author Seshavatharam is indebted to professors shri M. Nagaphani Sarma, Chairman, shri K.V. Krishna Murthy, founder Chairman, Institute of Scientific Research in Vedas (I-SERVE), Hyderabad, India and Shri K.V.R.S. Murthy, former scientist IICT (CSIR), Govt. of India, Director, Research and Development, I-SERVE, for their valuable guidance and great support in developing this subject.

\section{References}

[1] Frank Wilczek. QCD made simple (PDF). Physics Today. 53 (8): 22-28 (2000)

[2] M. Bojowald. Quantum cosmology: a review. Rep. Prog. Phys. 78 (2015) 023901 
[3] Hawking, S.W. Particle Creation by Black Holes. Communications in Mathematical Physics, 43, 199-220(1975)

[4] K. Tennakone, Electron, muon, proton, and strong gravity. Phys. Rev. D, 10, 1722 (1974)

[5] C. Sivaram and K. Sinha, Strong gravity, black holes, and hadrons. Physical Review D., 16(6), 1975-1978 (1977)

[6] De Sabbata V and M. Gasperini. Strong gravity and weak interactions. Gen. Relat. Gravit. 10, 9, 731-741, (1979)

[7] Salam A, Sivaram C. Strong Gravity Approach to QCD and Confinement. Mod. Phys. Lett., v. A8(4), 321-326 (1993)

[8] Roberto Onofrio. On weak interactions as shortdistance manifestations of gravity. Modern Physics Letters A 28, 1350022 (2013)

[9] Seshavatharam UVS and Lakshminarayana S. A Practical Model of Godel-Planck-Hubble-Birch Universe. Athens Journal of Sciences- Vol.6, Issue 3, 211-230 (2019)

[10] Seshavatharam UVS, Lakshminarayana S. To confirm the existence of atomic gravitational constant. Hadronic Journal.34(4):379 (2011)

[11] Seshavatharam UVS, Lakshminarayana S. Molar electron mass and the basics of TOE. Journal of Nuclear and Particle Physics. 2(6):132 -141 (2012)

[12] Seshavatharam UVS et al. Understanding the constructional features of materialistic atoms in the light of strong nuclear gravitational coupling. Materials Today: 3/10PB, Proceedings 3, 39763981 (2016)

[13] Seshavatharam UVS and Lakshminarayana S. Towards a workable model of final unification. International Journal of Mathematics and Physics 7(1), 117-130 (2016)

[14] Seshavatharam UVS and Lakshminarayana S. Understanding the basics of final unification with three gravitational constants associated with nuclear, electromagnetic and gravitational interactions. Journal of Nuclear Physics, Material Sciences, Radiation and Applications 4(1),1-19 (2017)

[15] Seshavatharam UVS and Lakshminarayana S. On the role of 'reciprocal' of the strong coupling constant in nuclear structure. Journal of Nuclear Sciences, 4( 2), 31-44 (2017).

[16] Seshavatharam UVS and Lakshminarayana S. Applications of gravitational model of possible final unification in both large and small scale physics. Prespacetime journal, Vol 7, issue2, 405-421 (2016)

[17] Seshavatharam UVS and Lakshminarayana S. A virtual model of microscopic quantum gravity, Prespacetime Journal, 9(1), 58-82 (2018)
[18] Seshavatharam UVS and Lakshminarayana S. On the role of four gravitational constants in nuclear structure. Mapana Journal of Sciences, 18(1), 2145 (2019)

[19] Seshavatharam UVS and Lakshminarayana S. On the Role of Large Nuclear Gravity in Understanding Strong Coupling Constant, Nuclear Stability Range, Binding Energy of Isotopes and Magic proton numbers - A Critical Review. J. Nucl. Phys. Mat. Sci. Rad. A. 6(2), 142-160 (2019)

[20] Seshavatharam UVS and Lakshminarayana S. On the Role of Squared Neutron Number in Reducing Nuclear Binding Energy in the Light of Electromagnetic, Weak and Nuclear Gravitational Constants - A Review. Asian Journal of Research and Reviews in Physics, 2(3): 1-22, (2019)

[21] Seshavatharam UVS and Lakshminarayana S. Super Symmetry in Strong and Weak interactions. Int. J. Mod. Phys. E, Vol.19, No.2, p.263-280 (2010).

[22] Seshavatharam UVS and Lakshminarayana S. SUSY and strong nuclear gravity in (120-160) $\mathrm{GeV}$ mass range. Hadronic journal, Vol-34, No 3, 277 (2011)

[23] Seshavatharam UVS and Lakshminarayana S. Integral charge SUSY in Strong nuclear gravity. Proceedings of the DAE Symp. on Nucl. Phys. 56, 842 (2011).

[24] Seshavatharam UVS and Lakshminarayana S. Molar Electron Mass and the Basics of TOE, Journal of Nuclear and Particle Physics, Vol. 2 No. 6, 132 (2012).

[25]F. Englert and R. Brout. Broken Symmetry and the Mass of Gauge Vector Mesons. Physical Review Letters, vol. 13, Issue 9, pp. 321-323 (1964)

[26] Higgs P. Broken Symmetries and the Masses of Gauge Bosons. Physical Review Letters. 13 (16): 508-509 (1964)

[27] The ATLAS Collaboration. Observation of a new particle in the search for the Standard Model Higgs boson with the ATLAS detector at the LHC. Phys.Lett. B716, 1-29 (2012)

[28] M. Tanabashi et al. (Particle Data Group), Phys. Rev. D 98, 030001 (2018)

[29] Niels Bohr. On the Constitution of Atoms and Molecules, Part I (PDF). Philosophical Magazine. 26 (151): 1-24 (1913)

[30] Seshavatharam UVS and Lakshminarayana S. Is Reduced Planck's Constant-An Outcome of Electroweak Gravity? Preprints 2019, $2019120231 \quad$ (doi: 10.20944/preprints201912.0231.v2)

[31] Seshavatharam UVS and Lakshminarayana S. On the role of Newtonian gravitational constant in 
estimating Proton-Electron mass ratio and baryon mass spectrum. International Journal of Innovative Studies in Sciences Engineering Technology. Vol 5, Issue 10, 18-21 (2019)

[32] Seshavatharam UVS and Lakshminarayana S. Hypothetical Role of Large Nuclear Gravity in Understanding the Significance and Applications of the Strong Coupling Constant in the Light of Up and Down Quark Clusters. Preprints 2019, 2019110398

(doi: 10.20944/preprints201911.0398.v2).

[33] T. Bayram, S. Akkoyun, S. O. Kara and A. Sinan, New Parameters for Nuclear Charge Radius Formulas, Acta Physica Polonica B. 44( 8), 17911799 (2013)

[34] Seshavatharam UVS and Lakshminarayana S. 4G Model of Fractional Charge Strong-Weak Super Symmetry. Preprints 2019, 2019120391 (doi: 10.20944/preprints201912.0391.v1)

[35]Brdar Vedran et al. X-Ray Lines from Dark Matter Annihilation at the keV Scale. Phys.Rev.Lett. 120 (2018) no.6, 061301

[36]A. Boyarskyet al. Surface brightness profile of the $3.5 \mathrm{keV}$ line in the Milky Way halo. arXiv:1812.10488v2 (2019)

[37] Nico Cappelluti et al. Searching for the $3.5 \mathrm{keV}$ Line in the Deep Fields with Chandra: The $10 \mathrm{Ms}$ Observations. The Astrophysical Journal, 854:179 (12pp) (2018)

[38] RW Pattie Jr et al. Measurement of the neutron lifetime using a magneto-gravitational trap and in situ detection. Science 11, Vol. 360, Issue 6389, pp. 627-632 (2018)

[39]F. E. Wietfeldt et al. A path to a $0.1 \mathrm{~s}$ neutron lifetime measurement using the beam method. Physics Procedia 51, 54 - 58 ( 2014 )

[40] Seshavatharam UVS and Lakshminarayana S. Neutron life time enigma in the light of Electromagnetic and Weak gravitational constants. Nucleus-2019, Dubna, Russia, Book of abstracts, Page 242 (2019)

[41] https://en.wikipedia.org/wiki/Atomic_radii_of_th e_elements_(data_page)

[42] https://openstax.org/books/astronomy/pages/18-2measuring-stellar-masses

[43]C.T. Russell and M.K. Dougherty. Magnetic Fields of the Outer Planets. Space Sci Rev, 152: 251-269 (2010)

[44]B. Canuel et al. Exploring gravity with the MIGA large scale atom interferometer. Science reports, 8:14064 (2018)

[45] Christos Merkatas et al. Shades of dark uncertainty and consensus value for the Newtonian constant of gravitation. arXiv:1905.09551v1 (2019) 